\title{
Chronic Fatigue Syndrome and Seasonal Affective Disorder: Comorbidity, Diagnostic Overlap, and Implications for Treatment
}

\author{
Michael Terman, PhD, Susan M. Levine, MD, Jiuan S. Terman, PhD, Sean Doherty, AB, \\ New York, New York
}

This study aimed to determine symptom patterns in patients with chronic fatigue syndrome (CFS), in summer and winter. Comparison data for patients with seasonal affective disorder (SAD) were used to evaluate seasonal variation in mood and behavior, atypical neurovegetative symptoms characteristic of SAD, and somatic symptoms characteristic of CFS. Rating scale questionnaires were mailed to patients previously diagnosed with CFS. Instruments included the Personal Inventory for Depression and SAD (PIDS) and the Systematic Assessment for Treatment Emergent Effects (SAFTEE), which catalogs the current severity of a wide range of somatic, behavioral, and affective symptoms. Data sets from 110 CFS patients matched across seasons were entered into the analysis. Symptoms that conform with the Centers for Disease Control and Prevention (CDC) case definition of CFS were rated as moderate to very severe during the winter months by varying proportions of patients (from $43 \%$ for lymph node pain or enlargement, to $79 \%$ for muscle, joint, or bone pain). Fatigue was reported by $92 \%$. Prominent affective symptoms included irritability (55\%), depressed mood (52\%), and anxiety (51\%). Retrospective monthly ratings of mood, social activity, energy, sleep duration, amount eaten, and weight change showed a coherent pattern of winter worsening. Of patients with consistent summer and winter ratings ( $n=73$ ), 37\% showed high global seasonality scores (GSS) $\geq 10$. About half this group reported symptoms indicative of major depressive disorder, which was strongly associated with high seasonality. Hierarchical cluster analysis of wintertime symptoms revealed 2 distinct clinical profiles among CFS patients: (a) those with high seasonality, for whom depressed mood clustered with atypical neurovegetative symptoms of hypersomnia and hyperphagia, as is seen in SAD; and (b) those with low seasonality, who showed

From the Department of Psychiatry, College of Physicians and Surgeons, Columbia University (MT); New York State Psychiatric Institute (MT, JST, SD); and the Department of Medicine, Beth Israel Medical Center (SML), New York, New York.

Support for this research was provided by grant RO1 MH42931 from the National Institute of Mental Health, and the Center for Environmental Therapeutics, Georgetown, Colorado.

Requests for reprints should be addressed to Michael Terman, PhD, Department of Psychiatry, Columbia University, 1050 Riverside Drive, Unit 50, New York, New York 10032 a primary clustering of classic CFS symptoms (fatigue, aches, cognitive disturbance), with depressed mood most closely associated with irritability, insomnia, and anxiety. It appears that a subgroup of patients with CFS shows seasonal variation in symptoms resembling those of SAD, with winter exacerbation. Light therapy may provide patients with CFS an effective treatment alternative or adjunct to antidepressant drugs. Am J Med. 1998;105(3A):115S-124S. (C) 1998 by Excerpta Medica, Inc.

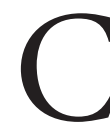
hronic fatigue syndrome (CFS) is characterized by sudden onset of persistent, debilitating fatigue and loss of energy that lasts $\geq 6$ months and cannot be attributed to other medical or psychiatric conditions. Prominent symptoms include headache; sore throat; pain in lymph nodes, muscles, and joints; and low-grade fever. ${ }^{1,2}$ Often cognition and behavior are impaired. The cause of illness remains elusive, although abnormal serologic, immunologic, and central nervous system (CNS) function have been implicated, ${ }^{3}$ and onset of the syndrome frequently appears to be triggered by a flulike infection.

Depression and its related symptoms (e.g., sleep disturbance, anxiety, and worsening of premenstrual symptoms) are also common in CFS. ${ }^{2}$ Some studies indicate that major depressive disorder is more prevalent in CFS than in other chronic medical illness. ${ }^{4,5}$ However, other studies have found that premorbid lifetime psychiatric disorder is similar in patients with CFS and the general population. $^{6}$

We have observed some CFS patients who complain of exacerbated mood state, diminished ability to work, and difficulty awakening during the winter months. Although by no means dominant in CFS, such a recurrent annual pattern is reminiscent of a particular major depressive subtype-seasonal affective disorder (SAD). Fatigue is a hallmark feature of SAD, as are the atypical neurovegetative symptoms of hypersomnia, appetite increase, and weight gain. ${ }^{7}$ In spring and summer, patients with SAD are free of depressive symptoms, although some become hypomanic. In winter, artificial-light therapy can also bring major improvement and even complete remission. ${ }^{8}$ 
The atypical symptom profile predicts successful clinical response to light. ${ }^{9}$

By strict diagnostic definition, CFS and SAD are mutually exclusive: the chronicity of CFS precludes remissions in spring and summer. Yet, winter exacerbation is common also in chronic and intermittent depression, and dysthymia, for which light therapy can be helpful at that time of year. Similarly, some patients with CFS show such a SAD-like overlay. If CFS and SAD are viewed as potentially comorbid disorders, one would expect to find certain symptoms common to both persisting yearround. Lam ${ }^{10}$ described 2 cases of comorbid CFS and SAD. Both patients showed an antidepressant response to light therapy, while one also showed improved arthralgia, a symptom associated with CFS. We, too, have treated several CFS patients with light, some with chronic hypersomnia or delayed sleep phase, ${ }^{11}$ others with prominent seasonal exacerbation of sleep symptoms, fatigue, and depressed mood. Some of these patients have shown marked improvement in their SAD-like symptoms, although without discernible effect on somatic symptoms such as fevers and respiratory ailments.

A recent study of patients with chronic or idiopathic fatigue, ${ }^{12}$ using a standard retrospective rating instrument for seasonal variation, ${ }^{13}$ found that nearly onequarter showed SAD-like patterns of mood, social activity, energy, sleep length, appetite, and weight gain. In the present study of CFS patients, we sought to (1) specify the frequency of somatic, behavioral, and affective symptoms in summer and winter; (2) compare the broad symptom profile with that of depressed SAD patients; (3) provisionally determine the frequency of a current or past-year major depressive episode (MDE); (4) gauge the monthly pattern and magnitude of seasonal symptom variation; and (5) compare the prevalence of winter worsening of atypical neurovegetative symptoms with that of SAD. If there were an identifiable subset of CFS patients with distinct winter worsening, light therapy might provide a viable treatment alternative or adjunct to drugs.

\section{PATIENTS AND METHODS}

\section{Subjects}

A total of 159 patients with CFS, all of whom met the revised Centers for Disease Control and Prevention (CDC) case definition, ${ }^{14}$ were selected from consecutive admissions to the practice of Susan M. Levine, MD. They were in her care for $\geq 6$ months at the time of the study. Patients with overlapping disorders-autoimmune disorders; metabolic conditions such as thyroid, diabetes, and adrenal insufficiency; chronic infections such as Lyme disease and human immunodeficiency virus-related illness; and primary affective disorders-were excluded. Although many patients experienced MDEs(see Results), none preceded the onset of CFS. In early sum- mer 1995, subjects were mailed a set of rating scale questionnaires (see Instruments), an explanatory cover letter, and a stamped return envelope, with up to 2 follow-up letters. The return rate was $80 \%(n=124)$. The respondents were again sent the identical survey in early winter 1995 , with a return rate of $92 \%(n=117)$. Seven cases were dropped from analysis because of age (outside the range of 18-65) or a move outside the geographic region, leaving a final matched-pairs sample of 110 , including 99 women (90\%) and 11 men (10\%), age $42.8 \pm 9.1$ years (mean $\pm \mathrm{SD}$ ). Duration of illness was $5.7 \pm 2.7$ years (range, 1-13 years). Most patients used medications and supplements, primarily: liver derivative complex (Kutapressin), $35 \%$; vitamin $\mathrm{B}_{12}, 35 \%$; vitamin C, $25 \%$; calcium, 20\%; magnesium, 19\%; levothyroxine sodium, 17\%; coenzyme Q10, 17\%; and gamma globulin, $17 \%$. The serotonin reuptake inhibitor antidepressants, fluoxetine and sertraline, were used by $12 \%$ and $11 \%$, respectively.

Results were compared with corresponding survey data for patients with $\mathrm{SAD},{ }^{15,16}$ including previously unpublished data, and a random sample population study of New York City adults. ${ }^{15,17}$ All groups were within the age range of $18-65$ years. SAD patients met DSM-III-R ${ }^{18}$ criteria for major depression, recurrent, or bipolar disorder not otherwise specified, both with seasonal pattern (winter type), and were selected from consecutive admissions to the outpatient Winter Depression Program of New York State Psychiatric Institute. They completed winter ratings while depressed and not using psychotropic medications, before entry into treatment studies.

\section{Instruments}

The Personal Inventory for Depression and SAD (PIDS) ${ }^{19}$ a 2-page self-rating questionnaire, includes 4 sections: (1) checklist for The Diagnostic and Statistical Manual of Mental Disorders, Fourth edition (DSM-IV) ${ }^{20}$ criteria for major depressive disorder, adapted from and scored according to the PRIME-MD ${ }^{21}$ algorithm; (2) 5 -point scale of magnitude of seasonal variation ("0", none; "4", extreme) in mood, social activity, energy, sleep duration, amount eaten, and body weight, with ratings summed as a global seasonality score ([GSS], 0-24 points), adapted from the Seasonal Pattern Assessment Questionnaire (SPAQ) $)^{13}$; (3) months of the year rated at seasonal extremes (e.g., highest, lowest) for the 6 component items of the GSS, adapted from the SPAQ; and (4) checklist for 9 atypical neurovegetative symptoms characteristic of winter depression.

The Systematic Assessment for Treatment Emergent Effects (SAFTEE) $)^{22}$ provides a 5-point rating scale ("1", absent; "3", moderate; " 5 ", very extreme) for past-week assessments of a wide-range of somatic, behavioral, and affective symptoms. It was originally designed to identify drug side effects using baseline and placebo control com- 
parisons. The list is categorized according to organ system and body part (e.g., head, eyes/vision, ears/hearing, mouth, chest, heart, stomach), and includes 88 core symptoms (with 8 additional items related to the menstrual cycle; not analyzed here), including most of those characteristic of CFS and SAD. (For tailoring to CFS patients, however, 5 additional items were added; Table 1, footnote $\boldsymbol{c}$ ). The SAFTEE is a comprehensive instrument that does not limit the symptom list to any specific syndromal or disease definition. Thus, various syndromal profiles — such as for CFS or SAD_ can be extracted statistically based on symptom frequencies, analyses of syndromal overlap and contrasts empirically derived. ${ }^{16}$

\section{RESULTS}

\section{Winter Symptomatology in CFS and SAD}

Table 1 lists symptoms rated moderate, severe, or very severe on the SAFTEE, in decreasing order of occurrence, for the winter survey of CFS patients. Eighteen symptoms occurred in more than half the patients; the 3 most prominent were fatigue, muscle/bone/joint pain, and thought/ concentration/memory problems. Other prevalent symptoms included insomnia, anxiety, and mood disturbance, as well as headaches, nasal congestion, and sore throat. The SAFTEE also served to identify a large set of symptoms that occurred only rarely (e.g., weight loss in only $10 \%$ of cases).

A comparison of CFS symptom frequencies with those of SAD provides an indication of overlap and contrasts between the syndromes. For example, whereas drowsiness is common in CFS and SAD alike (71\% and $67 \%$, respectively), sore throat is relatively uncharacteristic in SAD (53\% vs 12\%). Several relatively low-frequency CFS symptoms nevertheless appear to be more prevalent than in SAD (e.g., painful bowel movements, $20 \%$ vs 2\%). The "effect size of proportions" $(h)$ takes into account frequency of a symptom as well as the magnitude of group differences and provides a metric without the liabilities of multiple significance testing. ${ }^{23}$ As shown in Table 1, 18 symptoms showed large positive effect size $(h \geq 0.8)$, indicative of higher representation in CFS regardless of absolute frequency. For example, 53\% of CFS patients indicated light bothersome to eyes (photophobia), in comparison with only $6 \%$ of SAD patients $(h=1.15)$ - a very large difference. Using the criterion of small effect size $(h<0.3)$, CFS and SAD cannot be distinguished by drowsiness and hypersomnia; anxiety, irritability, and jumpiness/jitteriness; weight gain; and decreased sexual interest (and, in males, difficulty with erection). Where group differences were found, CFS patients were more symptomatic than SAD patients, with the exception of depressed $\operatorname{mood}(52 \%$ vs $86 \%, h=-0.77$ ).

\section{Seasonal Symptom Variation in CFS}

SAD patients are largely asymptomatic in summer, when CFS patients can remain quite ill. Furthermore, CFS "flare-ups" of acute physical illness are intermittent, and drug regimens tend to be continually modified, which can cast doubt on the generality of a single week's symptom profile. Indeed, CFS patients showed few clear seasonal contrasts in SAFTEE ratings, including those of depressive symptoms. Nasal bleeding and congestion were the only items to show dramatic winter exacerbation. Bleeding emerged or worsened in $20 \%$ of patients, whereas only $1.5 \%$ showed worsening in summer $(\mathrm{w}=0.79$, a very large effect size of McNemar's chisquare). ${ }^{23,24}$ Congestion-which occurred more frequently than bleeding-showed winter worsening in $36.9 \%$ of patients, and summer worsening in $13.8 \%$ $(\mathrm{w}=0.42$, which nears a large effect size $)$. Among other symptoms with predominant winter worsening - all with medium-to-small effect size-there was only 1 atypical symptom characteristic of SAD: weight gain $(32.3 \%$ winter worsening, $17.7 \%$ summer worsening, $\mathrm{w}=0.26)$. Several symptoms intensified in summer, with mediumto-large effect size: hemorrhoids (7.9\% winter worsening, $25.4 \%$ summer worsening, $\mathrm{w}=0.48)$; sweating $(15.6 \%$ winter worsening, $39.1 \%$ summer worsening, $\mathrm{w}=0.40)$; initial insomnia (15.6\% winter worsening, 36.9\% summer worsening, $\mathrm{w}=0.38)$; and feeling excited, overactive, or elated (20.6\% winter worsening, 32.8\% summer worsening, $\mathrm{w}=0.37)$. The latter 2 categories are consistent with summer hypomania, as is seen in a minority of SAD patients. ${ }^{9}$

Retrospective Ratings of Seasonal Contrasts. Figure 1 shows PIDS monthly ratings by CFS patients of mood, social contact, energy, sleep duration, amount eaten, and weight change. The ratings reflect cumulative judgments over years, by contrast with the 1-week "snapshot" ratings of the SAFTEE. The analysis is restricted to months judged consistently by each patient as best, worst, or neither, across the summer and winter surveys. As in SAD, there is evidence for distinct seasonal variation. The net relative frequencies of best and worst mood, for example, show a peak in May and June and a nadir in January and February. (There is also a secondary summer slump in July and August, as was previously found in the New York random-sample survey. ${ }^{15}$ Indeed, in August, $13.6 \%$ reported feeling best and $12.7 \%$ reported feeling worst, for a net relative frequency of $0.9 \%$ feeling best.) Social contact and energy level approximately parallel the annual mood cycle. Social contact shows a precipitous drop between December and January, with a net relative frequency of $27 \%$ socializing least-the most extreme of all the monthly ratings. Sleep duration, amount eaten, and weight gain show an inverse annual course, with winter maxima corresponding to the months of worst mood 
Table 1. Symptoms Reported in Winter Systematic Assessment for Treatment Emergent Effects (SAFTEE) Survey*

\begin{tabular}{|c|c|c|c|c|c|c|c|}
\hline \multirow[b]{2}{*}{ Symptom } & \multicolumn{2}{|c|}{$\begin{array}{l}\text { Proportion of } \\
\text { Patients }\end{array}$} & \multirow{2}{*}{$\begin{array}{l}\text { Effect } \\
\text { Size }^{\dagger}\end{array}$} & \multirow[b]{2}{*}{ Symptom } & \multicolumn{2}{|c|}{$\begin{array}{l}\text { Proportion of } \\
\text { Patients }\end{array}$} & \multirow{2}{*}{$\begin{array}{c}\text { Effect } \\
\text { Size }^{\dagger}\end{array}$} \\
\hline & CFS & SAD & & & CFS & SAD & \\
\hline Fatigue & 0.92 & 0.78 & 0.40 & Rapid heartbeat & 0.28 & 0.07 & 0.59 \\
\hline Muscle/bone/joint pain & 0.79 & 0.39 & 0.85 & Sweating & 0.28 & 0.04 & 0.74 \\
\hline \multirow{2}{*}{$\begin{array}{l}\text { Thought/concentration/ } \\
\text { memory problems }\end{array}$} & \multirow[t]{2}{*}{0.79} & \multirow[t]{2}{*}{0.56} & \multirow[t]{2}{*}{0.50} & Noise/ringing in ears & 0.28 & 0.05 & 0.66 \\
\hline & & & & Poor vision & 0.28 & 0.01 & 0.89 \\
\hline Drowsiness & 0.71 & 0.67 & 0.09 & Diarrhea & 0.27 & 0.13 & 0.37 \\
\hline Nasal congestion & 0.68 & 0.26 & 0.87 & Change in taste & 0.26 & 0.07 & 0.53 \\
\hline Middle insomnia & 0.66 & 0.20 & 0.97 & Difficulties with orgasm & 0.26 & 0.11 & 0.38 \\
\hline Headaches & 0.59 & 0.23 & 0.75 & Leg/arm swelling & 0.25 & 0.05 & 0.61 \\
\hline Gas & 0.59 & 0.30 & 0.59 & Breast tenderness & 0.25 & 0.04 & 0.67 \\
\hline Short-term memory problems ${ }^{\ddagger}$ & 0.58 & & & Eye swelling & 0.25 & 0.05 & 0.61 \\
\hline Initial insomnia & 0.56 & 0.25 & 0.66 & Chest pain & 0.25 & 0.04 & 0.66 \\
\hline Irritability & 0.55 & 0.65 & -0.21 & Difficulty swallowing & 0.23 & 0.01 & 0.79 \\
\hline Bloating $\ddagger$ & 0.53 & & & Earache & 0.22 & 0.00 & 0.98 \\
\hline Sore throat & 0.53 & 0.12 & 0.94 & Irregular heartbeat & 0.21 & 0.02 & 0.65 \\
\hline Light bothersome to eyes & 0.53 & 0.06 & 1.15 & Appetite decrease & 0.21 & 0.10 & 0.30 \\
\hline Depressed/“down”/“blue” & 0.52 & 0.86 & -0.77 & Painful bowel movements & 0.20 & 0.02 & 0.63 \\
\hline Hand/foot numbness & 0.51 & 0.10 & 0.97 & Change in stool color & 0.20 & 0.05 & 0.50 \\
\hline Anxiety & 0.51 & 0.52 & -0.02 & Shaking & 0.20 & 0.01 & 0.70 \\
\hline Unsteady on feet & 0.50 & 0.06 & 1.08 & Mouth sores & 0.19 & 0.03 & 0.51 \\
\hline Abdominal discomfort & 0.49 & 0.23 & 0.55 & Unwanted body movements & 0.18 & 0.01 & 0.67 \\
\hline Increased thirst & 0.46 & 0.19 & 0.60 & Hearing loss & 0.18 & 0.03 & 0.49 \\
\hline Late insomnia & 0.46 & 0.12 & 0.79 & Bleeding gums & 0.17 & 0.03 & 0.47 \\
\hline Dizziness/faintness & 0.44 & 0.09 & 0.82 & Laryngitis & 0.17 & 0.02 & 0.54 \\
\hline Lymph node pain/enlargement ${ }^{*}$ & 0.43 & & & Difficulty with erection ${ }^{\S}$ & 0.17 & 0.25 & -0.21 \\
\hline Fever/chills & 0.43 & 0.06 & 0.94 & Wheezing & 0.16 & 0.04 & 0.44 \\
\hline Restlessness & 0.42 & 0.17 & 0.57 & Overactive/excited/elated & 0.16 & 0.06 & 0.32 \\
\hline Dry mouth & 0.42 & 0.20 & 0.49 & Decreased urinary pressure & 0.15 & 0.01 & 0.57 \\
\hline Difficulty moving & 0.41 & 0.14 & 0.62 & Rigidity/stillness & 0.15 & 0.02 & 0.47 \\
\hline Skin rash/itch/irritation & 0.40 & 0.06 & 0.88 & Swollen/sore tongue & 0.14 & 0.02 & 0.47 \\
\hline Shortness of breath & 0.39 & 0.07 & 0.81 & Hemorrhoids & 0.13 & 0.05 & 0.30 \\
\hline Eye irritation & 0.39 & 0.09 & 0.72 & Nasal bleeding & 0.13 & 0.01 & 0.52 \\
\hline Frequent urination & 0.38 & 0.13 & 0.60 & Breast pain/discharge & 0.12 & 0.01 & 0.49 \\
\hline Finger swelling ${ }^{\ddagger}$ & 0.38 & & & Weight loss & 0.10 & 0.06 & 0.17 \\
\hline Constipation & 0.38 & 0.09 & 0.70 & Increased sexual interest & 0.10 & 0.10 & 0.01 \\
\hline Night sweats & 0.37 & & & Difficulty urinating & 0.10 & 0.00 & 0.65 \\
\hline Nausea & 0.36 & 0.06 & 0.80 & Change in urine color & 0.10 & 0.04 & 0.27 \\
\hline Hypersomnia & 0.35 & 0.45 & -0.19 & Genital swelling/discharge & 0.08 & 0.01 & 0.36 \\
\hline Decreased sexual interest & 0.33 & 0.48 & -0.29 & Excess salivation & 0.07 & 0.02 & 0.25 \\
\hline Bruising & 0.32 & 0.01 & 0.99 & Ear discharge & 0.07 & 0.00 & 0.55 \\
\hline Sunlight skin irritation & 0.31 & 0.00 & 1.19 & Genital discomfort & 0.07 & 0.00 & 0.54 \\
\hline Coughing & 0.31 & 0.10 & 0.52 & Double vision & 0.07 & 0.01 & 0.30 \\
\hline Dental problems & 0.31 & 0.02 & 0.87 & Vomiting & 0.06 & 0.00 & 0.47 \\
\hline Heartburn & 0.30 & 0.12 & 0.47 & Painful urination & 0.05 & 0.00 & 0.43 \\
\hline Appetite increase & 0.30 & 0.49 & -0.40 & Urinary burning & 0.05 & 0.00 & 0.43 \\
\hline Blurred vision & 0.30 & 0.02 & 0.84 & Excessive energy & 0.02 & 0.02 & -0.03 \\
\hline Weight gain & 0.29 & 0.29 & 0.01 & Loss of consciousness & 0.01 & 0.00 & 0.19 \\
\hline Jumpiness/jitteriness & 0.29 & 0.25 & 0.10 & Seizures & 0.00 & 0.00 & 0.00 \\
\hline
\end{tabular}

\footnotetext{
${ }^{*}$ Chronic fatigue syndrome $(\mathrm{CFS}), \mathrm{n}=110$; seasonal affective disorder $(\mathrm{SAD}), \mathrm{n}=86$. Symptom ratings of moderate to disabling.

${ }^{\dagger} h$ (directional); negative sign, SAD > CFS. $h=0.8$, large effect; $h=0.5$, medium effect; $h=0.3$, small effect.

* Symptom unassessed in SAD patients; addendum to the SAFTEE.

${ }^{\S}$ Males: CFS, $\mathrm{n}=11 ; \mathrm{SAD}, \mathrm{n}=20$.
} 

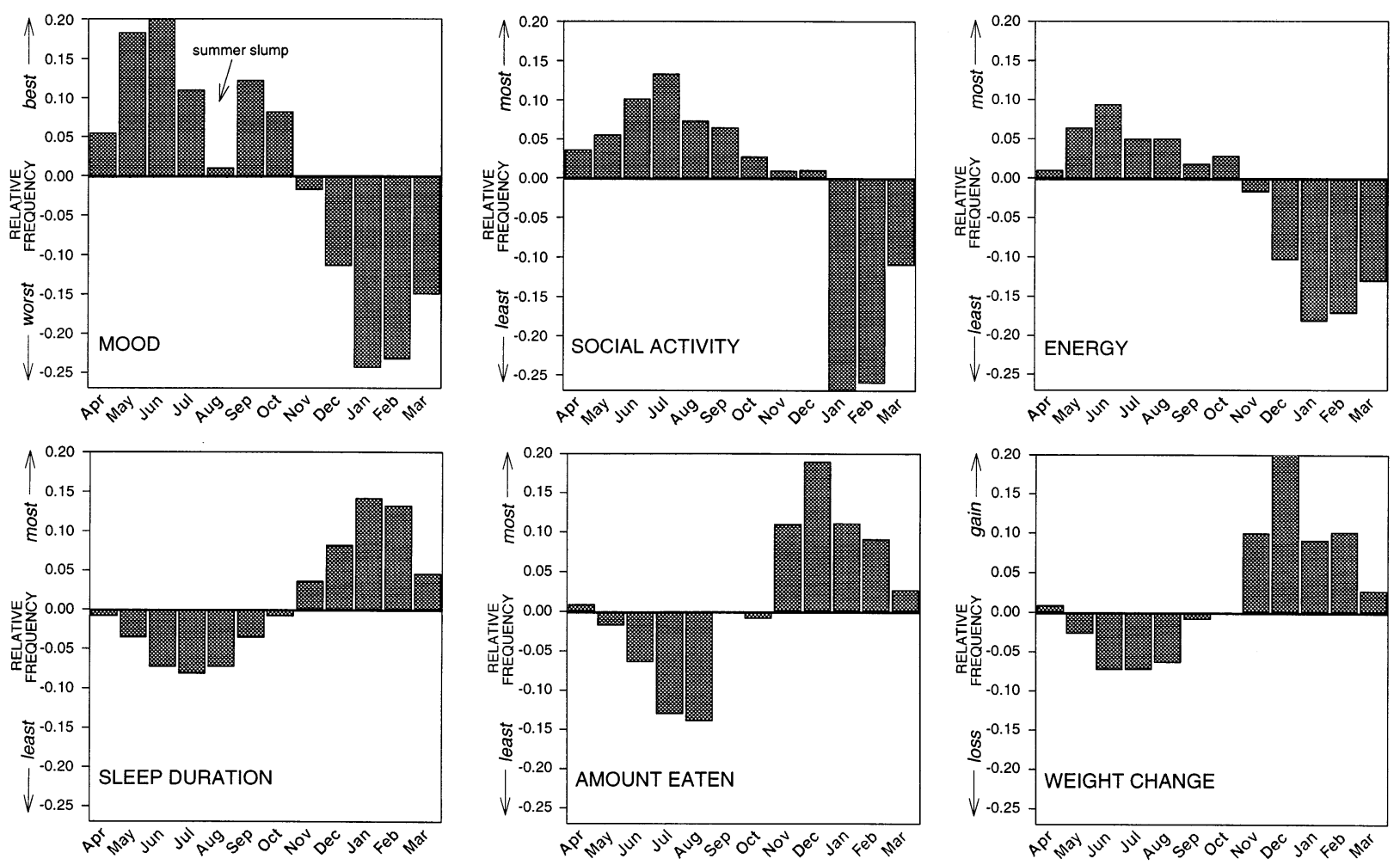

Figure 1. Retrospective ratings by months of the year, which show distinct variation in affective, behavioral, and physical factors. Patients separately identified "lowest" and "highest" months. Relative frequencies of lowest months were then subtracted from those of highest months, yielding the net relative frequency on the ordinate.

and least energy. Although most CFS patients do not experience distinct seasonal variation, the temporal pattern for the group as a whole mirrors that of SAD. ${ }^{17}$

Global Magnitude of Seasonal Variation in CFS and SAD Patients and the General Population. The PIDS estimate of seasonal change (GSS) is based on retrospective ratings that are expected to be consistent upon replication across the seasons.

CFS data were analyzed for the subset of 73 patients (66.4\%) that showed such consistency across winter and summer samples (GSS \pm 4 points, $\mathrm{r}=0.91, P<0.0001$ ), using the mean of the 2 estimates. (When all subjects were included, the correlation across samples was $r=0.59$ $[\mathrm{n}=110, P<0.0001]$, slightly lower than that previously reported for healthy subjects $[\mathrm{r}=0.65, \mathrm{n}=48$, $P<0.0001] .{ }^{25}$ ) The frequency distribution of seasonality scores (Figure 2) closely overlaps that of the New York random sample (mean \pm SD: $C F S, 8.1 \pm 5.5$; population, $7.2 \pm 3.6 ; t=1.31$, NS). The distribution for SAD patients is clearly separate, with a predominance of far higher scores (16.7 \pm 2.6$)$. However, $37 \%$ of the CFS patients-in comparison with $27 \%$ of the random sample $\left(\chi^{2}=5.8, \quad \mathrm{df}=1, \quad P=0.016\right)$ — showed seasonality scores $\geq 10$, indicative of discernible seasonality. Considering the winter survey alone, which matched the singlesample method used for the SAD patients and random sample, the total CFS group $(\mathrm{n}=110)$ showed even greater frequency of such high scores $(47 \%, \mathrm{n}=52)$.

\section{Features of Major Depressive Disorder in CFS}

The winter SAFTEE survey found $52 \%$ of CFS patients with current mood "depressed, down, or blue" (Table 1). In a more detailed examination of DSM-IV criteria for an MDE, the PIDS identified any such $\geq 2$-week interval(s) in the past year, irrespective of season (Table 2, footnote $\boldsymbol{b}): 51 \%$ of the group met MDE criteria on both summer and winter surveys, whereas only $26 \%$ indicated an absence of MDE. Table 2 lists the pertinent symptoms and their relative frequency. Approximately half the CFS group reported the 2 cardinal features of MDE, depressed mood or hopelessness $(45 \%)$ and anhedonia or loss of interest (50\%). Patients who met MDE criteria showed far higher rates ( $80 \%$ and $93 \%$, respectively), whereas those who did not showed far lower rates (3\% and $7 \%$ ).

Symptom frequencies within the depression cluster ranged widely. Anergy or fatigue was nearly ubiquitous, regardless of MDE status. (When this item was removed from the list of MDE criteria, $40 \%$ of patients still were considered to have shown clinical depression in the past year.) Similarly, insomnia or hypersomnia was reported by a large majority, but patients with MDE showed worsening $(h=0.71)$ relative to the others. By contrast, the remaining symptoms showed large differences $(h>0.90)$ 
between depressed and nondepressed patients. For example, poor concentration, and decrease or increase in appetite/weight, were nearly twice as prevalent given MDE.

\section{Seasonality and the Probability of Depression}

It is possible for an individual to show mood and behavioral disturbances specific to fall and winter that fall short of MDE criteria (i.e., subsyndromal SAD). Indeed, the population prevalence of subsyndromal SAD is far greater than that of SAD itself. ${ }^{26,27}$ There are additional groups (prevalence unknown) that do not experience depressed mood in winter, yet also show distinct seasonal variation of SAD-associated symptoms such as anergy, hypersomnia, and weight gain. Among 115 patients in an outpatient clinic for recurrent depression, $16 \%$ met criteria for $\mathrm{SAD}^{28}$; among a group of 79 psychiatric inpatients with major depression, $11 \%$ gave seasonality ratings within the range of SAD. Across all these patients, seasonality and depression ratings were correlated significantly $(\mathrm{r}=0.30, P<0.01) .^{29} \mathrm{SAD}$ is thus not predominant among depressives. Among a group of 303 primary care patients, 25\% reported winter-seasonal pain (a cardinal symptom of $\mathrm{CFS}^{14}$ ), whereas only $9 \%$ met criteria for SAD. ${ }^{30}$ However, $27 \%$ of the patients with pain showed concurrent major depression. In a study of 73 patients with CFS or idiopathic chronic fatigue, Zubieta and colleagues ${ }^{12}$ failed to detect an association between seasonality and depression ratings.

We assessed the degree of covariation of seasonality and MDE by selecting 58 patients who showed consistent global ratings (GSS \pm 4 points) and consistent MDE diagnosis on the 2 PIDS surveys. The group was divided into a $2 \times 2$ contingency table, by seasonality rating (strong, GSS $\geq 14, \mathrm{n}=12$, all with winters worse; weaker, GSS $<14, \mathrm{n}=46$ ) and MDE status (positive, $\mathrm{n}=37$; negative, $\mathrm{n}=21$ ). All strongly seasonal patients indicated MDE within the past year; by contrast only about half with weaker seasonality indicated MDE (25/46 [54.3\%]). Seasonality and recent depressive history were significantly associated $\left(\chi^{2}=8.59, \mathrm{df}=1, P=0.003\right)$. We reached the same conclusion when high and low seasonality scores were partitioned at $\geq 10$ and $<10$ according to the criteria of Zubieta and colleagues ${ }^{12}\left(\chi^{2}=4.19\right.$, $\mathrm{df}=1, P=0.04)$.

\section{Atypical Neurovegetative Symptoms in CFS and SAD}

The DSM-IV diagnostic algorithm for MDE does not distinguish between "typical" (or melancholic) and atypical symptomatology for sleep, appetite, and weight, each of which can increase or decrease. The SAFTEE survey (Table 1) found current ratings of insomnia to be more frequent in CFS than in SAD, although occurrence of hypersomnia was similar. Atypical symptoms are prevalent

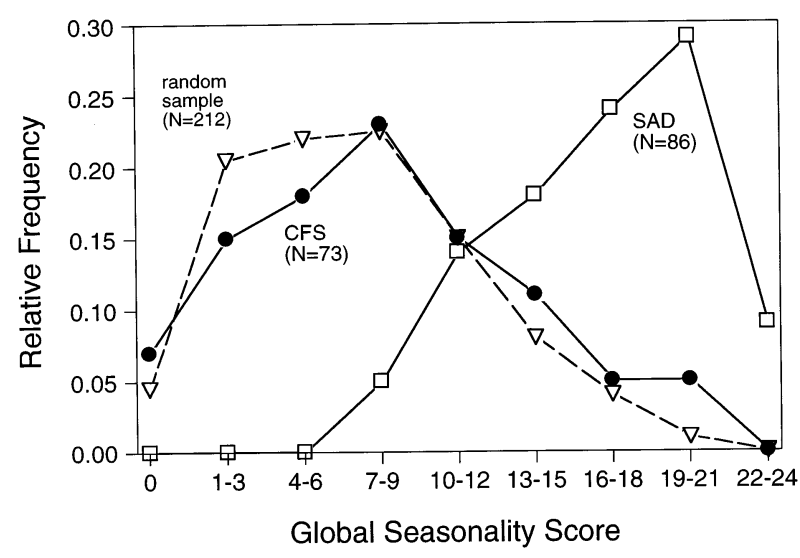

Figure 2. Relative frequency distributions of global seasonality scores (GSS) for 3 separate groups. Chronic fatigue syndrome (CFS) data are based on the mean GSS from summer and winter surveys, for patients whose scores remained within 4 points upon replication. Random sample data are from a general population study in New York City; data for diagnosed seasonal affective disorder (SAD) patients are from the Winter Depression Program at New York State Psychiatric Institute. $^{15}$

(but not exclusive) in SAD; their presence predicts the antidepressant response to light therapy. ${ }^{9}$ The PIDS probes specifically for past experience of winter worsen-

Table 2. Symptoms of Major Depressive Episode (MDE) Within Past Year Among Patients with Chronic Fatigue Syndrome $^{*}$

\begin{tabular}{lccc}
\hline & $\begin{array}{c}\text { Whole } \\
\text { Group } \\
(\mathrm{n}=110)\end{array}$ & $\begin{array}{c}\mathrm{MDE}^{\dagger} \\
(\mathrm{n}=56)\end{array}$ & $\begin{array}{c}\text { No } \\
\mathrm{MDE}^{\ddagger} \\
(\mathrm{n}=29)\end{array}$ \\
\hline $\begin{array}{l}\text { Anmptom Class } \\
\text { Insomnia or hypersomnia }\end{array}$ & 0.97 & 1.00 & 0.90 \\
$\begin{array}{l}\text { Poor concentration } \\
\text { Appetite/weight decrease }\end{array}$ & 0.72 & 0.93 & 0.66 \\
$\quad$ or increase & 0.68 & 0.86 & $0.45^{\S}$ \\
$\begin{array}{l}\text { Anhedonia or loss of } \\
\quad \text { interest }\end{array}$ & 0.50 & 0.93 & $0.45^{\S}$ \\
$\begin{array}{l}\text { Depressed mood or } \\
\quad \text { hopelessness }\end{array}$ & 0.45 & 0.80 & $0.07^{\S}$ \\
$\begin{array}{l}\text { Fidgetiness or slowness } \\
\text { Self-reproach or guilt }\end{array}$ & 0.41 & 0.66 & $0.03^{\S}$ \\
$\quad \begin{array}{l}\text { Suicidal thoughts or } \\
\quad \text { ideation }\end{array}$ & 0.38 & 0.64 & $0.03^{\S}$ \\
& & 0.27 & $0.00^{\S}$
\end{tabular}

\footnotetext{
* Proportion of group indicating symptom class on both summer and winter surveys.

${ }^{\dagger}$ Patients met diagnostic criteria if $\geq 5$ symptom classes were indicated (including $\geq 1$ anhedonia/loss of interest, and depressed mood or hopelessness) on both summer and winter surveys.

${ }^{\ddagger}$ Patients who failed to meet criteria on both summer and winter surveys.

${ }^{\S}$ Large effect size $(0.90 \leq h \leq 2.07)$, MDE vs No MDE.
} 
Table 3. Atypical Symptoms Rated as "Distinctly Worse in Winter"*

\begin{tabular}{lcccc}
\hline & & \multicolumn{3}{c}{ Chronic Fatigue Syndrome } \\
\cline { 3 - 5 } & $\begin{array}{c}\text { Seasonal affective } \\
\text { disorder } \\
\text { Symptom }\end{array}$ & $\begin{array}{c}\text { Whole group } \\
(\mathrm{n}=84)\end{array}$ & $\begin{array}{c}\text { High seasonals } \\
\left.{ }^{\dagger}=110\right) \\
(\mathrm{n}=52)\end{array}$ & $\begin{array}{c}\text { Low } \\
\text { seasonals } \\
\text { GSS }<10 \\
(\mathrm{n}=58)\end{array}$ \\
\hline Hypersomnia & 0.77 & 0.45 & 0.67 & $0.24^{\ddagger}$ \\
Difficulty awakening & 0.85 & 0.50 & 0.79 & $0.24^{\ddagger}$ \\
Anergy/fatigue & 0.88 & 0.57 & 0.79 & $0.38^{\ddagger}$ \\
Evenings worse & 0.40 & 0.34 & 0.46 & $0.22^{\S}$ \\
Afternoon slump & 0.69 & 0.52 & 0.67 & $0.38^{\S}$ \\
Carbohydrate craving & 0.77 & 0.60 & 0.77 & $0.45^{\S}$ \\
Carbohydrate ingestion & 0.70 & 0.46 & 0.69 & $0.26^{\ddagger}$ \\
Sweet cravings in PM & 0.58 & 0.50 & 0.67 & $0.35^{\S}$ \\
Weight gain & 0.82 & 0.47 & $0.62^{\|}$ & $0.34^{\S}$ \\
\hline
\end{tabular}

GSS $=$ global seasonality scores.

* Proportion of cases with positive ratings in winter surveys. ${ }^{\dagger}$ Global seasonality score. ${ }^{*}$ Large effect size $(0.86 \leq h \leq 1.16)$, high vs low seasonals. ${ }^{\S}$ Medium effect size $(0.51 \leq h \leq 0.67)$, high vs low seasonals. "Medium effect size $(h=0.47)$, high seasonals vs SAD patients.

ing in a large set of atypical neurovegetative symptoms (Table 3); it does not, however, assess mood reactivity and rejection sensitivity, which are among the DSM-IV criteria for depressive disorders "with atypical features."

As shown in Table 3, CFS patients who reported high seasonal variation showed consistently higher frequency of atypical symptoms (46-79\%) than those with low seasonal variation (22-45\%). Furthermore, those with high seasonality showed atypical symptom frequencies within the range of SAD patients, with only one exception: reports of winter weight gain were significantly more common in $\mathrm{SAD}$ ( $82 \%$ vs $62 \%, \chi^{2}=7.1, \mathrm{df}=1, P=0.008$, $h=0.47$ [a medium effect size]).

\section{Clustering of CFS and SAD Symptoms}

Intercorrelations among symptoms may differ for CFS patients who do or do not show a seasonal pattern. To illustrate such differences, we used a hierarchical clustering method based on Euclidean distances and the complete linkage method (farthest neighbor) ${ }^{31}$ Figure 3 displays results for the 10 symptoms reported most frequently on the SAFTEE in the winter by CFS and SAD patients. ${ }^{16}$ Cases were included if the seasonality score was consistent across the winter and summer surveys ( \pm 4 points; GSS $\geq 10, \mathrm{n}=27$; GSS $<10, \mathrm{n}=46$ ). Three symptoms - fatigue, drowsiness, and thought/memory/ concentration problems (labelled as "cognitive problems")—-were highly prevalent in both groups. Highest frequency symptoms unique to the CFS group included muscle/bone/joint pain (labelled as "aches"), nasal congestion, middle insomnia, headaches, gas, short-term memory problems, and initial insomnia (Table 1). Those unique to the SAD group included depressed mood, hypersomnia, appetite increase, weight gain, anxiety, irritability, and decreased sexual interest (labelled as "decreased libido").
The tree diagram for CFS patients with high seasonality reveals 2 primary groupings of symptoms: a core cluster (including weight gain, appetite increase, hypersomnia, depressed mood, and decreased libido) typifying $\mathrm{SAD}$, and a secondary cluster in which a set of CFS-typical symptoms abuts the core. Other symptoms of SAD outside the atypical neurovegetative spectrum - anxiety and irritability - were more closely associated with insomnia and headache than with the primary core cluster. By contrast, the diagram for CFS patients with low seasonality shows a core cluster of the most prominent symptoms of CFS (including cognitive problems, fatigue, drowsiness, and aches). A secondary cluster is divided into 2 segments, with atypical neurovegetative symptoms (appetite, weight gain, and hypersomnia) located farthest from the primary core cluster. Depressed mood itself falls away from the atypical symptom cluster; rather, it is most closely associated with irritability, anxiety, and insomnia, which form a cluster adjacent to the CFS core.

\section{DISCUSSION}

CFS patients rated the current status of 93 somatic, behavioral, and affective symptoms, using a questionnaire version of the SAFTEE including 5 supplemental items characteristic of the syndrome, thus providing a symptom frequency profile of this clinical population. The highest-frequency symptoms, all rated moderate to very severe, had close parallels in the CDC case definition ${ }^{14}$ (fatigue, 92\%; muscle/bone/joint pain, 79\%; thought/ concentration/memory problems, $79 \%$ ); $8 \%$ of patients rated fatigue as absent or mild. With the exception of postexertional fatigue, which was not assessed except at original diagnosis, all CDC criterion symptoms were shown by $\geq 43 \%$ of the group (lymph node pain or en- 
largement showing the lowest frequency). However, several other items overlapped this range and thus also characterized the patients, including affective symptoms (irritability, 55\%; depressed mood, 52\%; anxiety, $51 \%$ ) and somatic complaints (nasal congestion, 68\%; gas, 59\%; bloating, 53\%; photophobia, $53 \%$; hand/foot numbness, $51 \%$; loss of steadiness on feet, $50 \%$; increased thirst, $46 \%$; and dizziness or faintness, $44 \%$ ).

A hierarchy of CFS symptom frequencies $\geq 30 \%$, generalized across past studies, ${ }^{2}$ shows extensive overlap with these SAFTEE data. For example, we found similar rates of constipation and diarrhea ( $38 \%$ vs $27 \%, h=0.24$, a negligible effect size), whereas constipation (also 38\% in our survey) was not mentioned in the cross-center summary. ${ }^{2}$ Whether some high-frequency symptoms represent side effects of medication, are secondary to other symptoms (e.g., photophobia-associated headache), or are also typical of non-CFS populations, is uncertain. However, the SAD comparison group showed marked contrasts, with far lower frequency of muscle/bone/joint pain, nasal congestion, middle insomnia, sore throat, photophobia, numbness, loss of steadiness, and dizziness/faintness (Table 1). On the other hand, the CFS and SAD groups were closely matched in drowsiness, irritability, and anxiety.

Our study was designed to determine the extent of seasonal variation, with winters worse, in CFS. Seasonality scores for CFS patients (GSS, 8.1 \pm 5.5 ) were similar to that of the general population sample (7.2 \pm 3.6$)$, and far lower than that of SAD patients $(16.7 \pm 2.6)$, who, however, were selected for high seasonality. Zubieta and associates $^{12}$ reported a group seasonality score of $6.7 \pm 5.7$ in 73 patients with CFS or idiopathic chronic fatigue, which closely agrees with our results. However, the low scores should not detract from the observation that a distinct subset of CFS patients-37\% in our study, $22 \%$ in that of Zubieta's group-shows pronounced seasonality with winters worse. A question is whether this reflects comorbid SAD (i.e., with symptom variation restricted primarily to the affective and atypical neurovegetative dimensions), or whether the somatic symptoms of CFS also show winter exacerbation. We found that patients with highest seasonality were significantly more likely to have experienced a recent or current MDE, using DSM-IV criteria. (By contrast, Zubieta's group did not detect a relation between seasonality and depression.) Furthermore, we found that atypical neurovegetative symptomatology was highly similar in SAD patients and CFS patients with high seasonality. Notably, winter hypersomnia occurred in $67 \%$ of seasonal CFS cases; carbohydrate craving in $77 \%$; and worsening of fatigue in $80 \%$.

The SAFTEE, however, when administered in contrasting seasons, showed little prospective evidence for winter emergence or worsening of SAD-like symptoms except for weight gain, whereas SAD patients register dis-

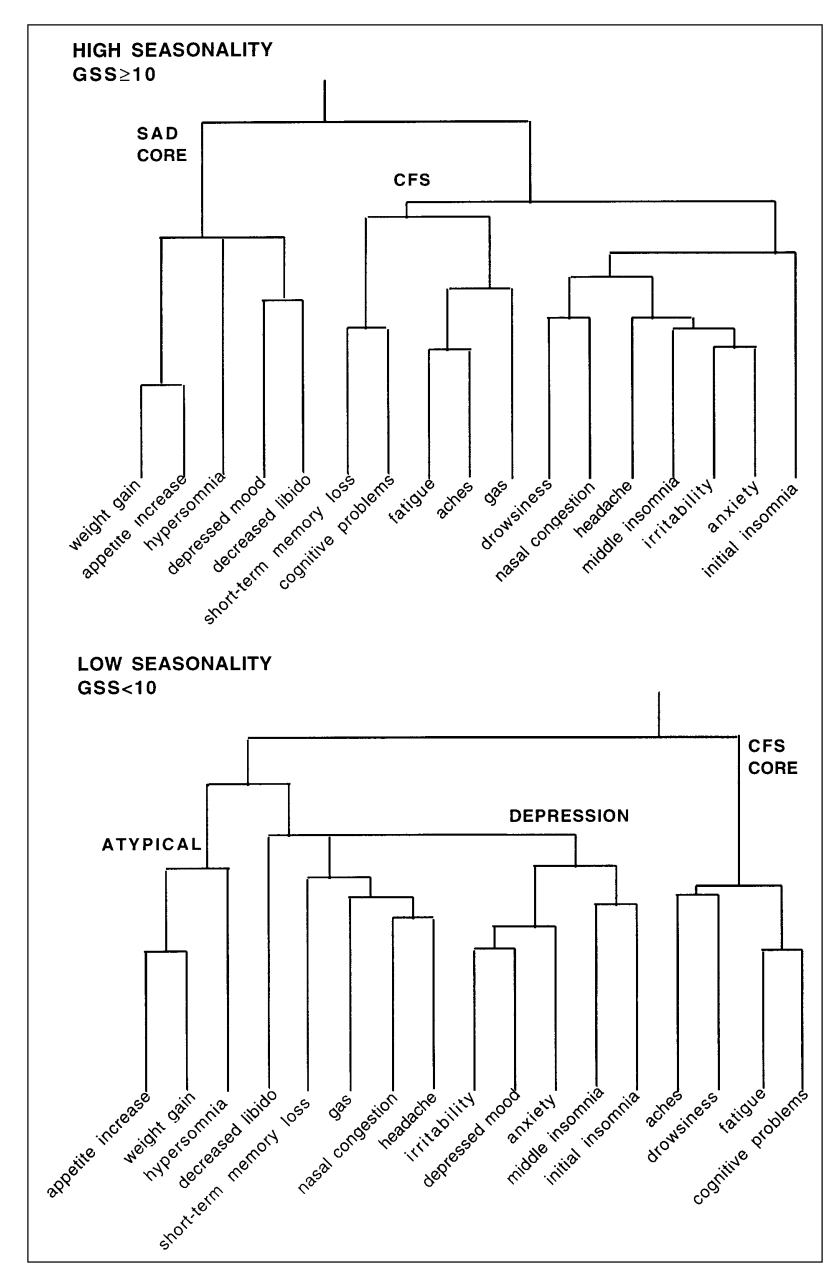

Figure 3. Tree diagrams illustrating hierarchical clustering of symptoms for CFS patients who rated their seasonality as high (GSS $\geq 10 ; n=27$ ) or low (GSS $<10$; $\mathrm{n}=46$ ). The symptoms comprise the 10 most frequently reported in winter by patients with chronic fatigue syndrome (CFS), and the 10 most frequently reported by patients with seasonal affective disorder $(\mathrm{SAD})^{16}$; given 3 overlapping symptoms (fatigue, drowsiness, and thought/concentration/memory problems), the list contains 17 items.

tinct changes, given their complete remissions in spring. ${ }^{16}$ There are several reasons that seasonal variation may be masked in the prospective SAFTEE ratings. Subsets of CFS patients may be chronically depressed or show summertime exacerbation. ${ }^{32}$ Furthermore, the use of antidepressants by about one-quarter of the patients may blunt affective symptoms (SAD patients in our studies have been unmedicated). By contrast, retrospective PIDS ratings draw on past years' cumulative experience, which may heighten the detectability of seasonal variation. Possibly, SAFTEE ratings made in the spring would reveal stronger contrasts with winter than did our summer ratings, which coincided with the months of summer slump 
(Figure 1). However, our SAFTEE ratings of nasal symptoms - congestion and bleeding - did show a large winter exacerbation, greatly exceeding that of SAD patients. Thus, the seasonal course of nasal symptoms should be viewed as characteristic of CFS, and distinct from the SAD-like variations seen in CFS patients with high seasonality scores.

CFS has been well recognized as inherently heterogeneous. For example, whereas some patients are depressed, others lack any diagnosable psychiatric disorder. ${ }^{33}$ Although antidepressants are frequently prescribed for CFS patients, clinical trials have met with mixed success. ${ }^{34,35}$ Interestingly, a recent open trial of sertraline led to major improvement in atypical neurovegetative symptoms-hypersomnia, appetite increase, and carbohydrate craving-regardless of the presence of depressed mood. $^{36}$

The hierarchical cluster analyses (Figure 3) reveal 2 distinct clinical subgroups within CFS, patients with high or low seasonal variation. Given high seasonality, depressed mood is most closely associated with a primary cluster of atypical neurovegetative symptoms. Given low seasonality, depressed mood is most closely associated with symptoms of agitation and anxiety. There is an apparent parallel in a recent analysis of 2 clinical subgroups with $\mathrm{SAD}^{9}$ : the primary group (83\%) was comprised of patients who responded to light therapy, and for whom depression was most closely associated with atypical symptoms; the second group (17\%) was comprised of nonresponders to light therapy, for whom depression was most closely associated with melancholic features (e.g., anxiety and guilt). Responders and nonresponders alike showed close association of depression and fatigue; fatigue itself was the least predictive symptom of therapeutic response to light. By contrast, the strongest positive predictors were atypical symptoms of hypersomnia, afternoon or evening slump, reverse diurnal variation (evenings worse) and carbohydrate craving — not all of which are assessed by the SAFTEE.

The potential utility of light therapy in CFS is underscored by our finding of a SAD-like subgroup with atypical symptoms, and several treatment successes in case studies. ${ }^{10,11}$ This nonpharmacologic procedure may have an advantage over antidepressants, especially given the multiple medication regimen of many CFS patients. An algorithmic decision tree for light and drug administration in SAD, based on professional consensus, has recently been published. ${ }^{37}$ Clinical response tends to be rapid (within 2 weeks), so alternative treatments need not be substantially delayed. Light therapy also has been used successfully with several CFS patients who experience chronic, nonseasonal early insomnia and difficulty awakening (delayed sleep phase syndrome), indicative of circadian rhythm disturbance. ${ }^{11,38}$ Homebound CFS patients may routinely receive inadequate daily light expo- sure, which may precipitate depressive and atypical neurovegetative symptoms regardless of the season; some may benefit by year-round light therapy. ${ }^{39}$ Indeed, the fact that patients with premorbid histories of affective disorder were excluded from our study suggests that a by-product of CFS - increased time indoors - may predispose some patients to disorders of light deprivation including SAD, subsyndromal SAD, and chronic depression with atypical features.

Drawbacks to conventional bright light therapy include the time required for daily exposure, and potential photophobic responses and eyestrain (Table 1). ${ }^{40,41}$ Glare disturbance can be controlled by use of overhead lighting devices and selective filtering of short-wavelength light. ${ }^{42} \mathrm{~A}$ current investigational treatment using far lower light intensity presents a diffuse, gradually rising signal to sleeping patients, which simulates an outdoor dawn. ${ }^{39,43}$ Both morning bright light and dawn simulation therapies phase-advance endogenous circadian rhythms (e.g., nocturnal melatonin secretion), which may be key to both sleep adjustment and antidepressant action. ${ }^{38}$ Clinical trials are needed to determine whether this treatment approach can be applied in CFS to alleviate seasonal exacerbation of affective or physical symptoms (or both), and whether treatment efficacy is confined to the fall/winter season.

\section{REFERENCES}

1. Holmes GP, Kaplan JE, Glancz NM, et al. Chronic fatigue syndrome: a working case definition. Ann Intern Med. 1988; 108:387-389.

2. Komaroff AL, Buchwald D. Symptoms and signs of chronic fatigue syndrome. Rev Infect Dis. 1991;13:S8-S11.

3. Bell DS. Chronic fatigue syndrome update: findings now point to CNS involvement. Postgrad Med. 1994;96:73-81.

4. Kruesi JP, Dale J, Straus SE. Psychiatric diagnoses in patients who have chronic fatigue syndrome. J Clin Psychiatry. 1989;50:53-56.

5. Katon WJ, Buchwald DS, Simon GE, et al. Psychiatric illness in patients with chronic fatigue and those with rheumatoid arthritis. J Gen Intern Med. 1991;6:277-285.

6. Hickie I, Lloyd A, Wakefield D, et al. The psychiatric status of patients with the chronic fatigue syndrome. Br J Psychiatry. 1990;156:534-540.

7. Rosenthal NE, Sack DA, Gillin JC, et al. Seasonal affective disorder: a description of the syndrome and preliminary findings with light therapy. Arch Gen Psychiatry. 1984;41: 72-80.

8. Terman M, Terman JS, Quitkin FM, et al. Light therapy for seasonal affective disorder: a review of efficacy. Neuropsychopharmacology. 1989;2:1-22.

9. Terman M, Amira L, Terman JS, et al. Predictors of response and nonresponse to light treatment for winter depression. Am J Psychiatry. 1996;153:1423-1429.

10. Lam RW. Seasonal affective disorder presenting as chronic fatigue syndrome. Can J Psychiatry. 1991;316:680-682.

11. Terman M. Light therapy. In: Micozzi M, ed. Fundamentals of Complementary and Alternative Medicine. London and New York: Churchill Livingstone, 1996:149-159.

12. Zubieta JK, Engleberg NC, Yargic LI, et al. Seasonal symp- 


\section{A Symposium: Chronic Fatigue Syndrome/Terman et al}

tom variation in patients with chronic fatigue: comparison with major mood disorders. J Psychiatr Res. 1994;28:1322.

13. Rosenthal NE, GH Bradt, Wehr TA. Seasonal Pattern Assessment Questionnaire. Bethesda, MD: National Institute of Mental Health, 1987.

14. Fukuda K, Straus SE, Hickie I, et al. The chronic fatigue syndrome: a comprehensive approach to its definition and study. Ann Intern Med. 1994;121:953-959.

15. Terman M. On the question of mechanism in phototherapy for seasonal affective disorder: considerations of clinical efficacy and epidemiology. J Biol Rhythms. 1988;3:155172.

16. Terman M. On the specific action and clinical domain of light treatment. In: Lam RW, ed. Seasonal Affective Disorder and Beyond: Light Treatment of SAD and Non-SAD Conditions. Washington, DC: American Psychiatric Press, 1998:91-115.

17. Terman M, Botticelli SR, Link BG, et al. Seasonal symptom patterns in New York: patients and population. In: Thompson C, Silverstone T, eds. Seasonal Affective Disorder. London: Clinical Neuroscience, 1989:11-95.

18. American Psychiatric Association. Diagnostic and Statistical Manual of Mental Disorders, 3rd ed.-revised (DSM-IIIR). Washington, DC: American Psychiatric Association, 1987.

19. Terman M, Williams JBW. Personal Inventory for Depression and SAD (PIDS). New York: New York State Psychiatric Institute, 1993.

20. American Psychiatric Association. Diagnostic and Statistical Manual of Mental Disorders, 4th ed. (DSM-IV). Washington, DC: American Psychiatric Association, 1994.

21. Spitzer RL, Williams JBW, Kroenke K, et al. Utility of a new procedure for diagnosing mental disorders in primary care: the Prime-MD 1000 study. JAMA. 1994;273:1749-1756.

22. National Institute of Mental Health. Systematic Assessment for Treatment Emergent Effects (SAFTEE). Rockville, MD: National Institute of Mental Health, 1986.

23. Cohen J. Statistical Power Analysis for the Behavioral Sciences. Hillsdale, NJ: Erlbaum, 1988.

24. Siegel S. Nonparametric Statistics for the Behavioral Sciences. New York: McGraw-Hill, 1956.

25. Wirz-Justice A. The Seasonal Pattern Assessment Questionnaire (SPAQ): some comments. Light Treatment Biol Rhythms. 1993;5:31-32.

26. Kasper S, Wehr TE, Bartko JJ, et al. Epidemiological findings of seasonal changes in mood and behavior. Arch Gen Psychiatry. 1989;46:823-822.

27. Rosen LN, Targum SD, Terman M, et al. Prevalence of seasonal affective disorder at four latitudes. Psychiatry Res. 1990;31:131-144.
28. Thase ME. Comparison between seasonal affective disorder and other forms of recurrent depression. In: Rosenthal NE, Blehar MC, eds. Seasonal Affective Disorders and Phototherapy. New York: Guilford Press, 1989:64-78.

29. Kasper S, Kamo T. Seasonality in major depressed inpatients. J Affect Disord. 1990;19:243-248.

30. Schlager D, Froom J, Jaffe A. Winter depression and functional impairment among ambulatory primary care patients. Compr Psychiatry. 1995;36:18-24.

31. Statistics, version 5.2.1. Evanston, IL: SYSTAT, 1992.

32. Wehr TA, Giesen HA, Schultz PM, et al. Contrasts between symptoms of summer depression and winter depression. $J$ Affect Disord. 1991;23:173-183.

33. Krupp LB, Mendelson WB, Friedman R. An overview of chronic fatigue syndrome. J Clin Psychiatry. 1991;52:403410.

34. Goodnick PJ, Sandoval R. Psychotropic treatment of chronic fatigue syndrome and related disorders. J Clin Psychiatry. 1993;54:13-20.

35. Vercoulen JHMM, Swanink CMA, Zitman FG, et al. Randomized, double-blind, placebo-controlled study of fluoxetine in chronic fatigue syndrome. Lancet. 1996;347:858861.

36. Behan PO, Haniffah BAG, Doogan DP, et al. A pilot study of sertraline for the treatment of chronic fatigue syndrome. (Abstr.) Clin Infect Dis. 1994;18:S111.

37. Lam RW, Wirz-Justice A, Terman M. Light therapy for depressive disorders: indications and efficacy. In: Rush AJ, ed. Modern Problems in Pharmacopsychiatry: Clinical Decision Trees in the Pharmacotherapy of Mood Disorders. Basel: Karger, 1997:215-234.

38. Terman M. Light treatment. In: Kryger MH, Roth T, Dement WC, eds. Principles and Practice of Sleep Medicine, 2nd ed. Philadelphia: WB Saunders, 1993:1012-1029.

39. Terman M. Light on sleep. In: Schwartz WJ, ed. Sleep Science: Integrating Basic Research and Clinical Practice. Basel: Karger, 1997:230-251.

40. Potaznick W, Kozol N. Ocular manifestations of chronic fatigue and immune dysfunction syndrome. Optom Vis Sci. 1992;69:811-814.

41. Caffery BE, Josephson JE, Samek MJ. The ocular signs and symptoms of chronic fatigue syndrome. J Am Optom Assoc. 1991;65:187-191.

42. Remé CE, Rol P, Grothmann K, et al. Bright light therapy in focus: lamp emission spectra and ocular safety. Technol Health Care. 1996;4:403-413.

43. Terman M, Schlager D, Fairhurst S, et al. Dawn and dusk simulation as a therapeutic intervention. Biol Psychiatry. 1989;25:966-970. 